\title{
Microsatellite loci cross-species transferability in Aedes fluviatilis (Diptera:Culicidae): a cost-effective approach for population genetics studies
}

\author{
Laura Cristina Multini ${ }^{1}$, Mauro Toledo Marrelli ${ }^{1,2}$ and André Barretto Bruno Wilke $2^{2^{*}}$
}

\begin{abstract}
Background: Aedes fluviatilis is a neotropical mosquito species thought to be a potential vector of Yellow Fever viruses and can be infected with Plasmodium gallinaceum in laboratory. A better understanding of its genetic structure is very important to understand its epidemiologic potential and how it is responding to urbanization. The objective of this study was to survey the transferability of microsatellites loci developed for other Aedes to Ae. fluviatilis.

Findings: We tested in Ae. fluviatilis 40 pairs of primers known to flank microsatellite regions in Aedes aegypti, Aedes albopictus and Aedes caspius, and found eight loci that amplified consistently. The number of alleles per locus ranged from 2 to 15 , and the expected heterozygosity ranged from 0.09 to 0.85 .

Conclusions: We found that several microsatellite primers successfully transferred to Ae. fluviatilis. This finding opens avenues for cost-effective optimization of high-resolution population genetic tools.
\end{abstract}

Keywords: Aedes fluviatilis, Microsatellite, Culicidae, Genetic structure

\section{Findings}

\section{Background}

Aedes fluviatilis (Lutz, 1904) [1] is a neotropical species found in Central and South America [2]. These mosquitoes can be found in wild, semi wild, urbans and suburbans environments [3]. Females are highly anthropophilic, more active during the day but can also bite at night and have been observed blood feeding while already developing eggs [4]. Ae. fluviatilis mosquitoes are considered a potential vector of the yellow fever viruses, are naturally infected with Wolbachia (wFlu) and can be experimentally infected with Plasmodium gallinaceum [5-7].

Previous studies showed that Ae. fluviatilis is adapted to the urban environment $[8,9]$. Considering, the scarce information about this species biology and ecology, a better understanding of its genetic structure is important

\footnotetext{
* Correspondence: andrebw.br@gmail.com

${ }^{2}$ Department of Epidemiology, School of Public Health, University of São

Paulo, Av. Dr. Arnaldo 715, São Paulo, SP CEP-01246-904, Brazil

Full list of author information is available at the end of the article
}

to access its epidemiologic potential and how it is responding to increasing urbanization. However, no molecular markers are currently available for population genetic studies to better understand adaptation and selection processes affecting this culicid.

Microsatellites or Simple Sequence Repeats (SSR) are molecular markers commonly used for population genetics studies, they are repetitive non-codon DNA regions composed by 1 to 6 base pairs in tandem, present in eukaryotic and prokaryotic genomes and are very useful to analyze the genetic structure of mosquitoes $[10,11]$. However only a few species have specific designed microsatellite primers and their development is not a simple task, a fact that limits its utilization in a broader spectrum of species.

Transferability of microsatellite loci between close taxa have been successfully performed before [12, 13], reducing primer development cost encouraging studies of population genetic structure in species with no specific developed microsatellite loci. Our objective was to survey the transferability to Ae. fluviatilis of microsatellite 
Table 1 Microsatellite loci amplified in Aedes fluviatilis

\begin{tabular}{|c|c|c|c|c|c|c|}
\hline Locus & Sequences 5'-3' & Repetitive motif & Source species & $\mathrm{T}\left({ }^{\circ} \mathrm{C}\right)$ & Size range & References \\
\hline \multirow[t]{2}{*}{ OchcB5 } & F: GCTTCGAATCTTGATGAGCA & CAC & Ae. caspius & $52^{*}(55)$ & $194-196 *(155-157)$ & Porretta et al. [15] \\
\hline & R: TGGATGCAGAGTGTT TTGGA & & & & & \\
\hline \multirow[t]{2}{*}{ OchcB9 } & F: CCAAAACACTCTGCATCCAA & CTA & Ae. caspius & 55 & $90-288^{*}(272-282)$ & Porretta et al. [15] \\
\hline & R: GGACTGGCCGAATACAGAGA & & & & & \\
\hline \multirow[t]{2}{*}{ OchcD11 } & F: TTCGACTCAGTTCGACGAGA & GT & Ae. caspius & 55 & $88-132^{*}(135-148)$ & Porretta et al. [15] \\
\hline & R: GGTCAATTCGGTTGAGTGGTT & & & & & \\
\hline \multirow[t]{2}{*}{ Alb-tri-3 } & F: AGATGTGTCGCAATGCTTCC & AGA & Ae. albopictus & 56 & $93-453^{*}(123-153)$ & Beebe et al. [19] \\
\hline & R: GATTCGGTGATGTTGAGGCC & & & & & \\
\hline \multirow[t]{2}{*}{ Alb-tri-20 } & F: GTGCCGTTGATCATCCTGTC & GTG & Ae. albopictus & 56 & $105-120^{*}(165-201)$ & Beebe et al. [19] \\
\hline & R: TCCAGCACCGTGAGTAATCC & & & & & \\
\hline \multirow[t]{2}{*}{ Alb-tri-33 } & F: GGCTGCTGTTGTTGGTACG & GGC & Ae. albopictus & 56 & $102-219^{*}(137-182)$ & Beebe et al. [19] \\
\hline & R: CACGTTCAATCACCGGTTCC & & & & & \\
\hline \multirow[t]{2}{*}{ Alb-tri-44 } & F: CACTCGCGCGTGTTCTTC & CAC & Ae. albopictus & 56 & $165-180^{*}(173-212)$ & Beebe et al. [19] \\
\hline & R: GACGCACCATCAGCATCATC & & & & & \\
\hline \multirow[t]{2}{*}{ AEDC } & F: TGCAGGCCCAGATGCACAGCC & GTA & Ae. aegypti & 60 & $246-477^{*}(210-230)$ & Chambers et al. [17] \\
\hline & R: TCCGCTGCCGTTGGCGTGAAC & & & & & \\
\hline
\end{tabular}

$\mathrm{T}=$ annealing temperature; *Values found in Aedes fluviatilis. In parenthesis, size range found in original studies

primers previously developed for Aedes aegypti, Aedes albopictus and Aedes caspius.

\section{Methods}

Forty microsatellite primers originally designed for $A e$. aegypti, Ae albopictus or Ae. caspius were tested in nine Ae. fluviatilis' populations composed of 30 female mosquitoes each, comprising a total of 270 individuals, collected in urban parks throughout the city of São Paulo [8]. DNA samples were extracted using the DNeasy Blood and Tissue Kit (Qiagen, Hilden, Germany), following the manufacturers' protocol.

PCR reactions were performed as in Huber et al. [14], Porretta et al. [15], Porretta et al. [16], Chambers et al. [17], Delatte et al. [18], Beebe et al. [19] in an E6331000025 Eppendorf Thermocycler (Masterclycler Nexus Gradient, Eppendorf, Hamburg, Germany). A gradient PCR was performed to identify the ideal annealing temperature for primers that did not amplify with the developer's original protocol by testing identical PCR reactions across a range of annealing temperatures. Successful amplifications were size sorted in $1 \%$ agarose gels, stained with GelRed" dye (Biotium, Hayward, CA, USA) and examined under UV light.

After incorporation of the fluorescent dye (FAM, HEX and NED), PCR products were diluted (1:7) by mixing $3 \mu \mathrm{L}$ of each product with $21 \mu \mathrm{L}$ of ultra-pure water for a final volume of $30 \mu \mathrm{L}$. A second dilution was performed with $2 \mu \mathrm{L}$ of the previous dilution suspended in $8.925 \mu \mathrm{L}$ of formamide HIDI (Applied Biosystems,
Foster City, CA, USA) and $0.075 \mu \mathrm{L}$ of molecular weight marker GeneScan 500 ROX (Applied Biosystems, Foster City, CA, USA) for a final volume of $11 \mu \mathrm{L}$. The samples were sent to the Centro de Estudos do Genoma Humano da Universidade de São Paulo and processed in the automatic sequencer ABI 3730 (Applied Biosystems, Foster City, CA, USA). Fragment analyzes were performed on the software Gene Marker (v1.85 SoftGenetics, Centre County, State College, PA, USA). The allele number, observed heterozygosity $\left(H_{O}\right)$, expected heterozygosity $\left(H_{E}\right)$, deviations from Hardy-Weinberg equilibrium and assessment of linkage disequilibrium were calculated in Genepop (v4.2 http://genepop.curtin.edu.au/) [20] and Arlequin (v3.5) [21].

Table 2 Characterization of microsatellite loci in Aedes fluviatilis

\begin{tabular}{lllll}
\hline Locus & $\mathrm{N}_{\mathrm{a}}$ & $H_{O}$ & $H_{E}$ & $P$ \\
\hline OchcB5 & 2 & 0.00000 & 0.09248 & $\mathbf{0 . 0 0 0 0 0}$ \\
OchcB9 & 5 & 0.30224 & 0.37172 & $\mathbf{0 . 0 0 0 0 0}$ \\
OchcD11 & 4 & 0.92164 & 0.56264 & $\mathbf{0 . 0 0 0 0 0}$ \\
Albtri3 & 15 & 0.68605 & 0.85359 & 0.01953 \\
Albtri33 & 3 & 0.13704 & 0.14910 & 0.00495 \\
Albtri20 & 6 & 0.11236 & 0.11912 & 0.01130 \\
AEDC & 3 & 0.95880 & 0.50203 & $\mathbf{0 . 0 0 0 0 0}$ \\
Albtri44 & 11 & 0.89057 & 0.69567 & $\mathbf{0 . 0 0 0 0 0}$
\end{tabular}

All 270 individuals were analyzed with 8 microsatellite loci; $\mathrm{Na}=$ Number of alleles found in each locus; $H_{O}=$ Observed Heterozygosity; $H_{E}=$ Expected Heterozygosity; $P$ value of Hardy-Weinberg equilibrium, in bold significant values after Bonferroni correction 


\section{Results and discussion}

Our results show that several primers were successfully transferred to Ae. fluviatilis, which corroborates the results of Bello \& Becerra [12] that successfully used microsatellite primers developed for Ae. caspius in Ae. taeniorhynchus. The 8 microsatellite primer pairs that successfully transferred to Ae. fluviatilis (OchcB5, OchcB9, OchcD11, AEDC, Albtri-3, Albtri-20 Albtri-33 and Albtri-44) (Table 1) were found to be moderately polymorphic, which may not be surprising since microsatellite loci in Aedes mosquitoes are often less polymorphic than loci developed for other Culicidae genera (e.g. Culex quinquefasciatus and Anopheles funestus) [22-24].

The number of alleles ranged from 2 to 15 per locus per population, the allele sizes were different from the alleles found in original manuscripts, except for locus OchcB5. Although locus Albtri3 had a high number of alleles per locus in Ae. fluviatilis, two alleles were found most frequently in the populations examined. Tests for Hardy-Weinberg equilibrium were made for the 8 functional microsatellite loci (Table 2). The $H_{E}$ values were higher than the $H_{O}$ values in 5 of 8 conducted tests (Table 2). After 150 possible tests, linkage disequilibrium was found between the loci OchcB9 and Albtri20; OchcB9 and Albtri33; OchcD11 and Albtri3; Albtri33 and Albtri20; and OchcD11 and Albtri33, however none of the linkages were considered significant, once no two loci were consistently correlated across the tested mosquitoes. The highest level of polymorphism was found in locus Albtri3. In contrast OchcB5 was close to monomorphic and might not be useful for Ae. fluviatilis genetic structure studies although additional populations should be tested. Both Albtri20 and Albtri33 were monomorphic in one of the nine populations. The other loci were moderately polymorphic and potentially very useful in future population genetics analyses of this species.

Null alleles, which occur when mutations in the primer regions prevent primers from annealing, are commonly found in studies using microsatellite markers [23]. Their presence can lead to incorrect assumptions of low genetic diversity and possibly consequent overestimation of Wright's F-statistics values [25]. Although caution is recommended regarding the use of microsatellite loci that may have null alleles, as they are less informative and may be more ambiguous, they still could be included in the analysis if their bias do not influence the results significantly. [13, 23, 25]. Also, cross-species microsatellite loci transfer might result in an ascertainment bias, which means that rates of variability may be consistently lower in the new species than in the species for which the markers were developed. This may be erroneously interpreted as evolutionary dynamics fluctuations among species. However, this bias is drastically diminished when the target and original populations have distinct demographic patterns [26].

Cross-species microsatellite loci transferability from Ae. aegypti, Ae. albopictus and Ae. caspius to Ae. fluviatilis demonstrate that loci developed for a few species can be recast for population genetic studies of less well studied species. This finding may open a new range of genetic structure studies of poorly known and/or neglected mosquitoes, e.g. secondary epidemiological importance species as well as a cost-effective genetic population accessible tool.

\section{Competing interests}

The authors declare that they have no competing interests.

\section{Authors' contributions}

LCM, ABBW and MTM conceived and designed the experiments. LCM performed the experiments. LCM and ABBW analyzed the data. LCM, ABBW and MTM wrote the manuscript. All authors read and approved the final version of the manuscript.

\section{Acknowledgements}

The authors would like to thank the Fundação de Amparo à Pesquisa do Estado de São Paulo (FAPESP) for providing financial support (Grant 2013/ 15313-4). LCM is a fellow of FAPESP (2013/22347-2). ABBW is the recipient of a postdoctoral fellowship from FAPESP (2012/19117-2).

\section{Author details}

${ }^{1}$ Institute of Tropical Medicine of São Paulo, University of São Paulo, Av. Dr. Enéas de Carvalho Aguiar, 470, São Paulo, SP CEP 05403-000, Brazil. ${ }^{2}$ Department of Epidemiology, School of Public Health, University of São Paulo, Av. Dr. Arnaldo 715, São Paulo, SP CEP-01246-904, Brazil.

Received: 23 September 2015 Accepted: 10 December 2015 Published online: 15 December 2015

\section{References}

1. Wilkerson RC, Linton YM, Fonseca DM, Schultz TR, Price DC, Strickman DA. Making mosquito taxonomy useful: a stable classification of tribe Aedini that balances utility with current knowledge of evolutionary relationships. PLoS One. 2015;10(7):e0133602.

2. WRBU. Walter Reed Biosystematics Unit. Systematic Catalogue of Culicidae. 2013. http://www.wrbu.org/. Accessed 07 Oct 2013.

3. Cônsoli RAGB, Williams P, Moreno EC, Santos BS. Features of reproduction in laboratory reared Aedes fluviatilis (Lutz, 1904) (Diptera: Culicidade). Mem Inst Oswaldo Cruz. 1983;78:37-47.

4. Carvalho GC, Malafronte RS, Izumisawa CM, Teixeira RS, Natal L, Marrelli MT. Blood meal sources of mosquitoes captured in municipal parks in São Paulo, Brazil. J Vector Ecol. 2014;39:146-52.

5. Davis NC, Shannon RC. Studies on yellow fever in South America: attempts to transmit the virus with certain Aedine and Sabethine mosquitoes and with Triatomas (Hemiptera). Am J Trop Med Hyg. 1931;11:21-9.

6. de Camargo MV T, Cônsoli RAGB, Williams P, Krettli AU. Factors influencing the development of Plasmodium gallinaceum in Aedes fluviatilis. Mem Inst Oswaldo Cruz. 1983;78:83-94

7. Moreira LA, Iturbe-Ormaetxe I, Jeffery JA, Lu G, Pyke AT, Hedges LM, et al. A Wolbachia symbiont in Aedes aegypti limits infection with dengue, Chikungunya, and Plasmodium. Cell. 2009:139:1268-78.

8. Medeiros-Sousa AR, Ceretti-Jr W, Urbinatti PR, Carvalho GC, Paula MB, Fernandes A, et al. Mosquito fauna in municipal parks of São Paulo City, Brazil: a preliminary survey. J Am Mosq Control Assoc. 2013;29:275-9.

9. Ceretti-Júnior W, Medeiros-Sousa AR, Wilke ABB, Strobel RC, Orico LD, Teixeira RS, et al. Mosquito faunal survey in a central park of the city of São Paulo, Brazil. J Am Mosq Control Assoc. 2015;31:172-6.

10. Tóth G, Gáspári Z, Jurka J. Microsatellites in different eukaryotic genomes: survey and analysis. Genome Res. 2000;10:967-81. 
11. Edillo FE, Tripet F, McAbee RD, Foppa M, Lanzaro GC, Cornel AJ, et al. A set of broadly applicable microsatellite markers for analyzing the structure of Culex pipiens (Diptera: Culicidae) populations. J Med Entomol. 2007:44:145-9.

12. Bello F, Becerra V. Genetic variability and heterogeneity of Venezuelan equine encephalitis virus vector Ochlerotatus taeniorhynchus (Diptera: Culicidae) populations of the Colombian Atlantic coast, based on microsatellite loci. Genet Mol Res. 2009;8:1179-90.

13. Belisário CJ, Pessoa GCD, Santos PF, Dias LS, Rosa ACL, Diotaiuti L. Markers for the population genetics studies of Triatoma sordida (Hemiptera: Reduviidae). Parasit Vectors. 2015;8:269.

14. Huber K, Mousson L, Rodhain F, Failloux AB. Isolation and variability of polymorphic microsatellite loci in Aedes aegypti, the vector of dengue viruses. Mol Ecol Notes. 2001;1:219-22.

15. Porretta D, Bellini R, Urbanelli S. Characterization of microsatellite markers in the mosquito Ochlerotatus caspius (Diptera: Culicidae). Mol Ecol Notes. 2005;5:48-50.

16. Porretta D, Gargani M, Bellini R, Calvitti M, Urbanelli S. Isolation of microsatellite markers in the tiger mosquito Aedes albopictus (Skuse) Mol Ecol Notes. 2006;6:880-1.

17. Chambers EW, Meece JK, McGowan JA, Lovin DD, Hemme RR, Chadee DD, et al. Microsatellite isolation and linkage group identification in the yellow fever mosquito Aedes aegypti. J Hered. 2007;98:202-10.

18. Delatte H, Toty C, Boyer S, Bouetard A, Bastien F, Fontenille D. Evidence of habitat structuring Aedes albopictus populations in Reunion Island. PLoS Trop Dis. 2013;7:e2111.

19. Beebe NW, Ambrose L, Hill LA, Davis JB, Hapgood G, Cooper RD, et al. Tracing the tiger: population genetics provides valuable insights into the Aedes (Stegomyia) albopictus invasion of the Australasian Region. PLoS Negl Trop Dis. 2013;7(8):e2361.

20. Rousset F. Genepop'007: a complete reimplementation of the Genepop software for Windows and Linux. Mol Ecol Resour. 2008;8:103-6.

21. Excoffier L, Laval G, Schneider S. Arlequin ver. 3.0: an integrated software package for population genetics data analysis. Evol Bioinform Online. 2005;1:47-50.

22. Samb B, Dia I, Konate L, Ayala D, Fontenille D, Cohuet A. Population genetic structure of the malaria vector Anopheles funestus, in a recently re-colonized area of the Senegal River basin and human-induced environmental changes. Parasit Vectors. 2012;5:188.

23. Monteiro FA, Shama R, Martins AJ, Gloria-Soria, Brown JE, Powell JR. Genetic Diversity of Brazilian Aedes aegypti: Patterns following an Eradication Program. PLoS Negl Trop Dis. 2014;8:e3167.

24. Wilke ABB, Vidal PO, Suesdek L, Marrelli MT. Population genetics of neotropical Culex quinquefasciatus (Diptera: Culicidae). Parasit Vectors. 2014;7:468.

25. Carlsson J. Effects of microsatellite null alleles on assignment testing. J Hered. 2008;99(6):616-23.

26. Li B, Kimmel M. Factors influencing ascertainment bias of microsatellite allele sizes: impact on estimates of mutation rates. Genetics. 2013;195:563-72.

\section{Submit your next manuscript to BioMed Central and we will help you at every step:}

- We accept pre-submission inquiries

- Our selector tool helps you to find the most relevant journal

- We provide round the clock customer support

- Convenient online submission

- Thorough peer review

- Inclusion in PubMed and all major indexing services

- Maximum visibility for your research

Submit your manuscript at www.biomedcentral.com/submit

) Biomed Central 